\title{
pH Watch - Leveraging Pulse Oximeters in Existing Wearables for Reusable, Real-time Monitoring of $\mathrm{pH}$ in Sweat
}

\author{
Ananta Narayanan Balaji* \\ ananta@u.nus.edu \\ Dept. of Electrical \& Computer Engg. \\ National University of Singapore
}

\author{
Chen Yuan* \\ chenyuan@u.nus.edu \\ Dept. of Biomedical Engg. \\ National University of Singapore
}

\author{
Bo Wang \\ dcswbo@comp.nus.edu.sg \\ Dept. of Computer Science \\ National University of Singapore
}

\author{
Li-Shiuan Peh \\ peh@nus.edu.sg \\ Dept. of Computer Science \\ National University of Singapore
}

\author{
Huilin Shao \\ huilin.shao@nus.edu.sg \\ Dept. of Biomedical Engg. \\ National University of Singapore
}

\section{CCS CONCEPTS}

\begin{abstract}
Sweat is a readily accessible bodily fluid for detecting biomarkers such as $\mathrm{pH}$, glucose etc., enabling continuous and non-invasive assessment of the well-being of individuals. Our proposed work aims at leveraging pulse oximeter chips in current-day fitness trackers for real-time continuous monitoring of $\mathrm{pH}$ in sweat. We achieve that by fabricating a highly responsive and long-term reusable $\mathrm{pH}$ sweat sensor on a flexible material to achieve skin conformity, targeting the sensor to work at the reflected infrared $(880 \mathrm{~nm})$ and red (660nm) photoplethysmograph (PPG) signal intensities recorded by pulse oximeters. The sensor can be readily mounted atop any wearable with a pulse oximeter. We have successfully demonstrated a low-cost, low-power, highly-responsive and longterm reusable wrist-worn wearable prototype, $\mathrm{pH}$ Watch, for realtime continuous monitoring of $\mathrm{pH}$ value of sweat. We conducted on-body trials with 10 participants and $\mathrm{pH}$ Watch achieves an accuracy of $\approx 91 \%$. We also showed that the integration of our sweat sensor does not hinder the pulse oximeter from measuring heart rate and $\mathrm{SpO}_{2}$, and users can continue with their daily activities with motion artifacts removed efficiently from PPG signals using the TROIKA framework, resulting in heart rate and $\mathrm{SpO}_{2}$ measurements with an accuracy of $\approx 95 \%$ and $\approx 96 \%$ respectively when validated against commercial finger pulse oximeter measurements. To the best of our knowledge, $\mathrm{pH}$ Watch is the first demonstration of a reusable sweat sensor that can be readily integrated into today's smart watches with pulse oximeters, paving the way for ubiquitous sensing of biomarkers.
\end{abstract}

${ }^{*}$ Both authors contributed equally to this research.

\footnotetext{
Permission to make digital or hard copies of all or part of this work for personal or classroom use is granted without fee provided that copies are not made or distributed for profit or commercial advantage and that copies bear this notice and the full citation on the first page. Copyrights for components of this work owned by others than ACM must be honored. Abstracting with credit is permitted. To copy otherwise, or republish, to post on servers or to redistribute to lists, requires prior specific permission and/or a fee. Request permissions from permissions@acm.org.

MobiSys '19, June 17-21, 2019, Seoul, Republic of Korea

(C) 2019 Association for Computing Machinery.

ACM ISBN 978-1-4503-6661-8/19/06 ..\$15.00

https://doi.org/10.1145/3307334.3326105
}

\section{ACM Reference Format:} glucose, lactate etc.
- Human-centered computing $\rightarrow$ Ubiquitous and mobile devices; • Applied computing $\rightarrow$ Health care information systems.

\section{KEYWORDS}

Sweat sensor, $\mathrm{pH}$ sensing, Wearables, IoT

Ananta Narayanan Balaji, Chen Yuan, Bo Wang, Li-Shiuan Peh, and Huilin Shao. 2019. pH Watch - Leveraging Pulse Oximeters in Existing Wearables for Reusable, Real-time Monitoring of $\mathrm{pH}$ in Sweat. In The 17th Annual International Conference on Mobile Systems, Applications, and Services (MobiSys '19), June 17-21, 2019, Seoul, Republic of Korea. ACM, New York, NY, USA, 13 pages. https://doi.org/10.1145/3307334.3326105

\section{INTRODUCTION}

Most present day fitness trackers and smart watches measure critical health indicators such as heart rate, $\mathrm{SpO}_{2}$ concentration, sleep cycle etc. but they fail in their ability to track health indicators at the molecular level. This has attracted rapid research in the development of chemical sensors which can non-invasively measure analytes available in raw biofluids such as sweat, tears and urine. Of all the available raw bio-fluids, sweat can be obtained non-intrusively and readily, and thus is the most suitable choice for continuous real-time monitoring of indicators at the molecular level. In addition, sweat contains a rich amount of biomarkers such as sodium $\left(\mathrm{Na}^{+}\right)$, chloride $\left(\mathrm{Cl}^{-}\right)$, potassium $\left(\mathrm{K}^{+}\right)$, calcium $\left(\mathrm{Ca}^{2+}\right), \mathrm{pH}$,

Amongst the detectable bio-markers in sweat, $\mathrm{pH}$ plays a very important role in the diagnosis of many critical health conditions. Variations in $\mathrm{pH}$ value of skin can aid in the diagnosis of skin conditions such as dermatitis, acne and other skin infections [1]. It is also interesting to note that, under a dehydrated condition, there will be an increase in concentration of $\mathrm{Na}^{+}$in sweat which is indicated by a increase in the $\mathrm{pH}$ value of sweat [2], since the higher the sodium $\left(\mathrm{Na}^{+}\right)$levels in sweat, the higher the $\mathrm{pH}$ of sweat will be [3]. Also, an increase in perspiration rate triggers a rise in the $\mathrm{pH}$ value of sweat [2]. Hence, we can observe that the $\mathrm{pH}$ value of sweat provides information regarding the hydration level of our body, which is essential for fitness tracking as well as for diagnosing certain medical conditions. 
In recent years, pulse oximeters have been increasingly integrated into wrist-worn fitness trackers such as fitbit [4], garmin [5] to aid in the measurement of blood oxygen levels $\left(\mathrm{SpO}_{2}\right)$ with the help of the obtained photoplethysmograph (PPG) signals. There has also been increasing interest in finding critical health indicators using PPG signals obtained from pulse oximeters in fitness trackers. More recently, research has been carried out on measuring blood pressure [6] with the help of PPG signals in Empatica E4 wristband. Also, there have been attempts made on using fitness trackers for studying how human behavior impacts their health (bio-behavioral sensing) [7] with the help of bio-markers such as heart rate, $\mathrm{SpO}_{2}$ etc. measured by pulse oximeters along with behavior indicated by the corresponding activity patterns recorded by accelerometers.

With fitness trackers and smart watches increasingly ubiquitous, we propose to leverage the pulse oximeters in these wearables for sweat sensing, paving the way for enabling widespread deployment of molecular sensing. In this paper, we propose an approach for real-time monitoring of $\mathrm{pH}$ in sweat by leveraging pulse oximeters for $\mathrm{pH}$ sensing in sweat with the integration of a lowcost long-term reusable polyaniline (PANI) based $\mathrm{pH}$ sensing material. We demonstrate a low-power, highly responsive and longterm reusable wrist-worn wearable prototype, $\mathrm{pH}$-Watch, with integrated pulse oximeter and PANI sensor, for real-time continuous $\mathrm{pH}$ sensing from sweat. We have showed that mounting the PANI sensor on the pulse oximeter does not hinder the measurement of heart rate and $\mathrm{SpO}_{2}$ levels and motion artifacts can be removed efficiently from PPG signals during physical activities with TROIKA PPG motion removal framework [8] aiding in an accurate heart rate measurement from PPG signals. The proposed $\mathrm{pH}$ sensing approach can be readily integrated to current day smart watches with pulse oximeters.

We evaluated our sensor for its fundamental characteristics, namely sensitivity, repeatability, selectivity and reproducibility. We have also conducted on-body trials on human subjects and studied the efficiency of our approach in real time, continuous monitoring of $\mathrm{pH}$ from sweat and demonstrated how real-time $\mathrm{pH}$ measured by our prototype could be used to detect dehydration during exercise.

The rest of the paper is organized as follows: Section 2 discusses related work on $\mathrm{pH}$ sensing of sweat and throws light on the working principle behind pulse oximeters. Section 3 describes our sweat sensing approach. Section 4 evaluates our wearable prototype $\mathrm{pH}-$ Watch. This is followed by a discussion of the evaluation results in Section 5 and Section 6 concludes the paper.

\section{BACKGROUND AND RELATED WORKS}

\subsection{Sweat sensors}

A wearable provides a natural platform for real-time continuous sensing of sweat, as it is in constant contact with human skin [9][10]. The desired aspects of a wearable for sweat $\mathrm{pH}$ sensing are long-term reusability and low cost. Just as today's fitness trackers sense human heart rate continuously, repeatedly reusing the same low-cost wearable, sweat sensing on wearables should also be achievable on a low-cost, reusable wearable platform.
In this section, we first discuss prior research into wearable devices for real-time continuous monitoring of $\mathrm{pH}$ in sweat. They can be broadly classified into colorimetric sensing and electrochemical sensing approaches.

- Colorimetric sensing approach: Caldara et al. [11,12] implemented a low-power miniaturized smart textile based colorimetric $\mathrm{pH}$ sensor with highly sensitive electronics that measures $\mathrm{pH}$ based on color changes in the textile fabric treated with an organically modified silicate (ORMOSIL). This textile-based sensor can only be washed and reused for a short time of 15 days. Curto et al. [13] developed a real-time wearable, disposable microfluidic platform based on ionogels for colorimetric sensing of $\mathrm{pH}$ from sweat. While disposable sensors are particularly suited for some applications such as medical diagnostic tests, sweat $\mathrm{pH}$ sensing which is typically used in fitness and wellness applications is more fitting with a long-term reusable platform. Koh et.al [14] also reported a soft and thin microfluidic device for colorimetric sensing of multiple biomarkers - $\mathrm{pH}$, glucose, lactate and chloride in sweat. This device consists of sealed microfluidic channels for each biomarker of interest in sweat filled in with appropriate colorimetric reagents reacting with color changes to the concentration of corresponding biomarker of interest in sweat. However, this device requires chemical reagents to be replaced periodically, thereby making it non-reusable. Also, it cannot track time dependent changes in the concentration of biomarkers in sweat and hence, is unsuitable for real-time continuous sensing of $\mathrm{pH}$ in sweat.

- Electrochemical sensing approach: A wearable device based on cyclic voltammetry [15] has been reported for real-time continuous monitoring of $\mathrm{Ca}^{2+}$ and $\mathrm{pH}$ in sweat by an array of $\mathrm{Ca}^{2+}$ and $\mathrm{pH}$ ion selective electrodes (ISE) interfaced with a flexible PCB. Recently, a stretchable and skin attachable electrochemical $\mathrm{pH}$ sensor [16] has been developed for measuring glucose and $\mathrm{pH}$ from sweat with polyaniline based ISE for $\mathrm{pH}$ detection. However, these devices have a long-term stability of only 10 days and need to be replaced every 10 days, thus making them unsuitable for long-term reusability. Another recent work by Dang et al. [17] reported a wireless stretchable $\mathrm{pH}$ sensing system based on graphite polyurethane composite material along with RFID antenna and data processing as well as transmission circuitry for communication with smartphones. Although this device can be reusable up to 2 years, manufacturing of graphite polyurethane composite is very complex and requires expensive instrumentation. Our proposed PANIbased material, on the other hand, is low cost and requires no additional electronics, as pulse oximeters are commonplace in today's wearables.

- Other sensing approaches: A biosensing textile patch with fluidics handling and a $\mathrm{pH}$ sensitive dye with paired emitter-detector red LEDs [10] has been reported for continuous $\mathrm{pH}$ sensing. However, it suffers from non-reusability of the $\mathrm{pH}$ sensitive dye and requires complex sweat pumping methods to pump sweat into the $\mathrm{pH}$ sensor. Bandodkar et al. [18] combined tattoo-based sensor technology with potentiometry to develop an epidermal sensor for monitoring $\mathrm{pH}$ in sweat. The tattoo-based sensor consists of a $\mathrm{pH}$ sensitive ISE and a reference electrode facilitating potentiometric sensing of $\mathrm{pH}$ and is placed directly on the skin. However, as the name suggests, tattoo based approaches are not suitable for longterm reusability and can cause skin irritation if they remain on skin 


\begin{tabular}{lccc}
\hline Prior works & $\begin{array}{c}\text { Real-time and } \\
\text { continuous }\end{array}$ & $\begin{array}{c}\text { Long-term } \\
\text { reusability }\end{array}$ & $\begin{array}{c}\text { Compatibility with } \\
\text { fitness trackers }\end{array}$ \\
\hline$[10]$ & $\checkmark$ & $\times$ & $\times$ \\
{$[11,12]$} & $\checkmark$ & $\times$ & $\times$ \\
{$[13]$} & $\checkmark$ & $\times$ & $\times$ \\
{$[14]$} & $\times$ & $\times$ & $\times$ \\
{$[15]$} & $\checkmark$ & $\times$ & $\times$ \\
{$[16]$} & $\checkmark$ & $\times$ & $\times$ \\
{$[17]$} & $\checkmark$ & $\checkmark$ & $\times$ \\
{$[18]$} & $\checkmark$ & $\times$ & $\times$ \\
pH-Watch & $\boldsymbol{V}$ & $\mathbf{V}$ & $\mathbf{V}$ \\
\hline
\end{tabular}

Table 1: Comparison of prior works on wearable pH sensing vs. pH-Watch

for long. Table 1 summarizes the prior works on sweat $\mathrm{pH}$ sensing. We see long-term reusability and compatibility with existing wearables as features that can pave the way towards next-generation fitness trackers/smart watches with integrated sweat $\mathrm{pH}$ sensors.

In this paper, we have developed a low cost, reusable wrist worn pulse oximeter based prototype for real-time continuous sensing of $\mathrm{pH}$ value from sweat, which could be readily integrated into most existing fitness trackers. We also showed that our $\mathrm{pH}$ sensing approach can be easily interfaced with pulse oximeters without affecting their normal ability to measure heart rate and $\mathrm{SpO}_{2}$ levels in our body.

\subsection{Pulse oximeter and heart rate sensor}

As Figure 1 depicts, a pulse oximeter shines light onto the surface of the skin, detects the light intensity changes which is backscattered by the tissue and the blood, and then processes the signals to decode heart rate and/or $\mathrm{SpO}_{2}$.

The pulse oximeter is a sensor commonly used to measure heart rate and pulsatile oxygen saturation $\left(\mathrm{SpO}_{2}\right)$ level in a non-invasive fashion. It measures the concentration of $\mathrm{HbO}_{2}$ and $\mathrm{Hb}$ by checking their absorption coefficients of two wavelengths, one is usually in red light spectra $(660 \mathrm{~nm})$ and the other is usually in infrared light spectra (880nm or 940nm). Figure 2 depicts different absorption responses from $\mathrm{Hb}$ and $\mathrm{HbO}_{2}$ against the two wavelengths. When LED light illuminates red blood cells and vascular tissue bed, oxyhemoglobin absorbs less red components but more infrared components than deoxyhemoglobin. The oximeter then analyzes this absorption differentiation by sensing the intensity of either reflectance light or transmission light. The perceived light consists of pulsatile arterial blood component (known as AC) and non pulsatile arterial blood component (known as DC). Their ratio is then calculated using the following equation:

$$
R=\frac{R_{\text {red }}}{R_{\text {infra-red }}}=\frac{A C_{\text {red }} / D C_{\text {red }}}{A C_{\text {infra-red }} / D C_{\text {infra-red }}}
$$

This ratio will be further processed with empirical value to generate the final $\mathrm{SpO}_{2}$ output. The $\mathrm{AC}$ depicts pulse rate, thus enabling the pulse oximeter to also function as a heart rate monitor sensor.

While clinical instruments rely on the sensing of the intensity of transmission light, portable sensors such as that on smart watches (e.g. Samsung Gear) and fitness trackers (e.g. Fitbit Versa) use reflectance light-based sensors. The Maxim MAX30101 chip is a reflectance LED-based sensor which obviates the need for probes for transmission light sensing, thus enabling small footprint, ultra-low power operation and robust motion artifact resilience. It has been adopted in various wearable hardware, such as OpenHAK kit [21] and Hexiwear platform [22]. Figure 4 illustrates the system diagram. The chip has two major blocks, one optical, and the other electrical. The optical part integrates cover glass for optimal and robust performance. The electrical subsystem integrates red (peak at $660 \mathrm{~nm}$ ) and infra-red (peak at $880 \mathrm{~nm}$ ) LEDs to emit light, with LED drivers modulating the LED pulses for $\mathrm{SpO}_{2}$ measurements. The photodiode perceives reflective visible light as well as invisible light and converts them to an electrical signal proportional to the light intensity. The following 18-bit current ADC (analog-todigital converter) samples and converts the signal to digitized code with an ambient light cancellation (ALC) function. The signal contains the periodicity information of a pulse rate, which is named as photoplethysmogram (PPG). The ALC has an internal track/hold circuit to cancel ambient light noise from the reflectance light and improve dynamic range. Maxim 30101 system also contains an onchip temperature sensor to calibrate for temperature variations.

Figure 3 exhibits sample PPG waveforms across time, measured from human finger tips illuminated by red LED and infra-red LED, respectively. This is obtained with the Maxim 30101 evaluation board via USB interface and the plot graphed with its software GUI.

\section{PROPOSED WEARABLE SWEAT SENSOR}

\subsection{Overview of $\mathrm{pH}$ Watch}

We have developed a wrist-worn wearable prototype, $\mathrm{pH}$ Watch for real-time monitoring of $\mathrm{pH}$ value in sweat. The wristband was chosen as the form factor for $\mathrm{pH}$ Watch because of the popularity of wrist-worn wearables among users; We wish to show that our $\mathrm{pH}$ sensing approach could be easily extended to current-day wristworn fitness trackers with integrated pulse oximeters. Although we have adopted a wristband as our form factor, our prototype $\mathrm{pH}$ Watch could be easily modified as an arm band, chest strap or head band. $\mathrm{pH}$ watch can simultaneously monitor $\mathrm{pH}$ from sweat along with heart rate and $\mathrm{SpO}_{2}$ values in real-time. $\mathrm{pH}$ Watch is not only limited to athletes and can be used by any user who sweats during daily walking or running.

Figure 5 shows photos of our prototype $\mathrm{pH}$-Watch, which comprises (a) a pulse oximeter chip, Maxim's MAX30101, (b) a flexible $\mathrm{pH}$ sensor made with polyaniline (PANI) polymer that we designed and fabricated, mounted atop the MAX30101, (c) interfaced with an off-the-shelf wearable, Texas Instrument (TI)'s sensor tag CC2650 which runs the software algorithms for obtaining $\mathrm{pH}$, heart rate and $\mathrm{SpO}_{2}$, and (d) showing how our prototype is worn. The pulse oximeter emits infrared and red light which traverses through the PANI sensor and the human skin then reflects back through the tissue to the PANI sensor. The PANI sensor is fabricated to respond with increasing reflected infrared-to-red $(800 \mathrm{~nm}$ to $660 \mathrm{~nm})$ ratio to increasing $\mathrm{pH}$ values, hence the reflected light back to the pulse oximeter can be used to differentiate $\mathrm{pH}$ levels. The design, fabrication and properties of our PANI sensor are explained in Section 3.2. The MAX30101 pulse oximeter chip is connected to the TI CC2650, a low-power, wireless sensor tag which has 10 integrated sensors including accelerometer, gyroscope, equipped with an ARM Cortex-M3 processor and powered with a coin-cell battery. We chose the TI CC2650 as it is a highly extensible off-theshelf wearable platform. On the CC2650, we run the software algorithm for $\mathrm{pH}$ estimation from sweat, that is explained in Section 3.4, 


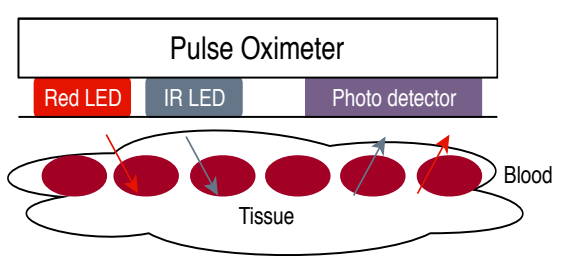

Figure 1: Illustration of pulse oximeter based on reflectance intensity sensing

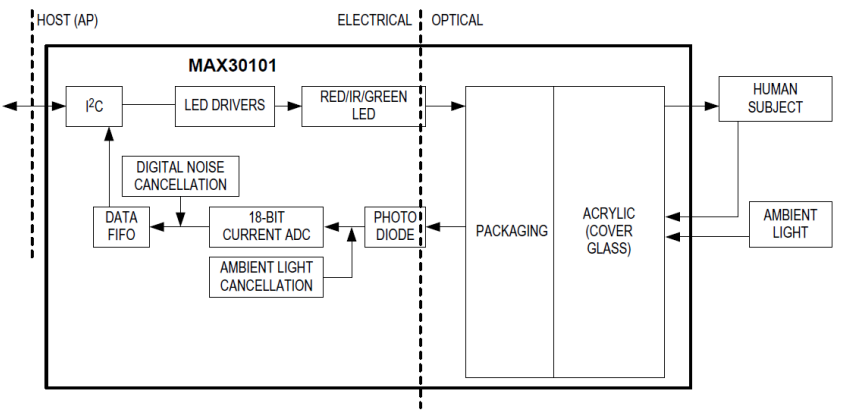

Figure 4: Max30101 system diagram [20]

as well as the software algorithm for obtaining the heart rate and $\mathrm{SpO}_{2}$ readings, what MAX30101 was originally designed to sense, discussed in Section 3.5. Section 3.6 details the software flow of $\mathrm{pH}-$ Watch.

Figure 6 presents the system-level block diagram of our entire $\mathrm{pH}-$ Watch system. The CC2650 comes with $128 \mathrm{~KB}$ flash to store our code and 20KB SRAM for data processing. CC2650 is connected to the TI debugger devpack which adds the ability to debug the sensor tag and also provides extended I/O pins for the sensor tag. In $\mathrm{pH}$ Watch, we use the SCL and SDA pins of the debugger devpack for inter-integrated circuit (I2C) communication between MAX30101 and CC2650. The CC2650 sensor tag is also connected to a low power TI watch devpack LCD display via SPI using the expansion header from debugger devpack which displays $\mathrm{pH}$ value of sweat, heart rate and $\mathrm{SpO}_{2}$ values in real-time. The ADC values of infrared and red light reflected from skin and blood are measured by MAX30101 and sent over the I2C bus connection to CC2650 sensor tag. Algorithms for $\mathrm{pH}$ estimation as well as heart rate and $\mathrm{SpO}_{2}$ estimation are run on the $\mathrm{CC} 2650$.

\subsection{Design, fabrication and evaluation of polyaniline (PANI) sensor}

While conventional glass-type electrodes have been widely used for $\mathrm{pH}$ measurement, for integration with wearables, they possess several disadvantages due to their size constraint, rigidity

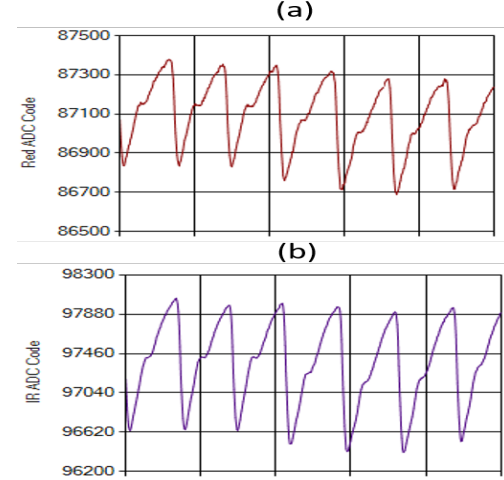

Figure 3: Sample (a) red (b) IR PPG waveforms measured on finger tip

and need for electrode maintanence. More recently, $\mathrm{pH}$-responsive polymers have been developed [23][24]. These polymers can be readily integrated with wearables due to their flexible nature, ease of miniaturization, and good biocompatibility. In particular, conductive polymer polyaniline (PANI) offers not only large-range and sensitive $\mathrm{pH}$ responsiveness, but also enables real-time optical readouts. Prior research has shown that after proton-mediated post-polymer doping and dedoping, PANI exhibits significant changes in the near-infrared spectrum, which is a target frequency of pulse oximeter [25][26][27]. We thus chose to develop a PANIbased $\mathrm{pH}$ sensor through mounting the polymer on the MAXIM pulse oximeter to enable real-time, reusable and continuous monitoring of sweat $\mathrm{pH}$. This integration offers the following advantages. First, the polymer acts as a dual matrix support and indicator dye which is a substance used to show visually the condition of a solution with respect to the presence of a particular material (such as different concentration of hydrogen ion) by change of color, and can be readily interfaced for safe skin contact. The polymer is biocompatible and does not disintegrate [26] [18] [28]; This improves long-term stability, satisfying our reusability design goal, as it eliminates any possible dye leaching, which is a common problem seen in sensors using other $\mathrm{pH}$-responsive small molecule dyes [29]. Second, when treated with different $\mathrm{pH}$ solutions, PANI shows a significant and rapid response at the wavelengths $660 \mathrm{~nm}$ and $880 \mathrm{~nm}$ - distinct wavelengths illuminated and measured by the pulse oximeter. This high compatibility for direct integration thus enables sensitive and reusable monitoring of sweat $\mathrm{pH}$. To the best of our knowledge, this development presents the first example on the integrated application of PANI with pulse oximeter, where PANI functions as an optical $\mathrm{pH}$ sensor and the pulse oximeter is the detector that can sensitively sense the resultant PANI changes in red and infra-red absorbance.

3.2.1 Working principle. As shown in Figure 7, the PANI film is placed atop the MAXIM pulse oximeter, which is an integrated light source and detector. When the PANI is in contact with acidic (low $\mathrm{pH}$ ) solution, the polymer becomes protonated, through the conversion of Emeraldine Base (EB) to Emeraldine Salt (ES). This 

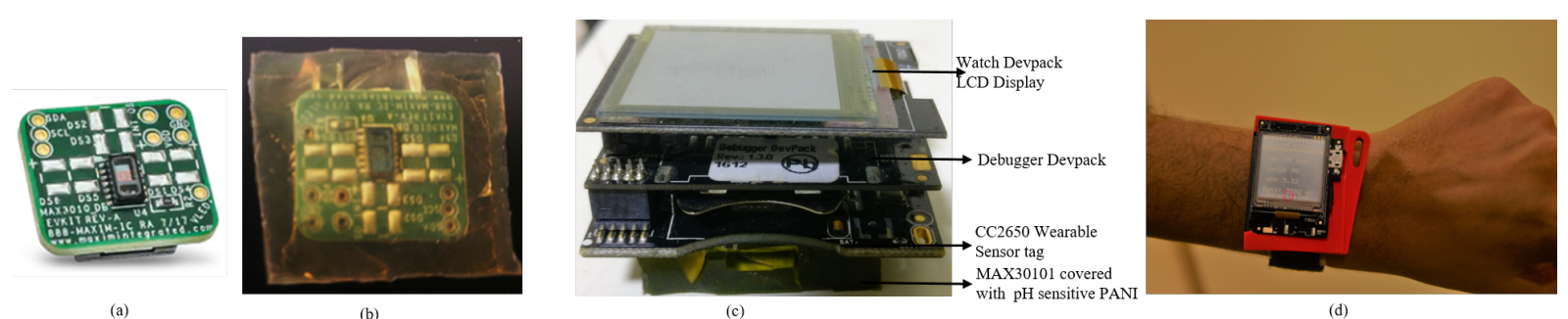

Figure 5: Various components of pH-Watch: (a) the MAX30101 pulse oximeter chip, (b) with a PANI-based flexible sensor mounted atop, (c) the entire $\mathrm{pH}$-watch prototype, and (d) the $\mathrm{pH}$ Watch with wrist strap worn on skin

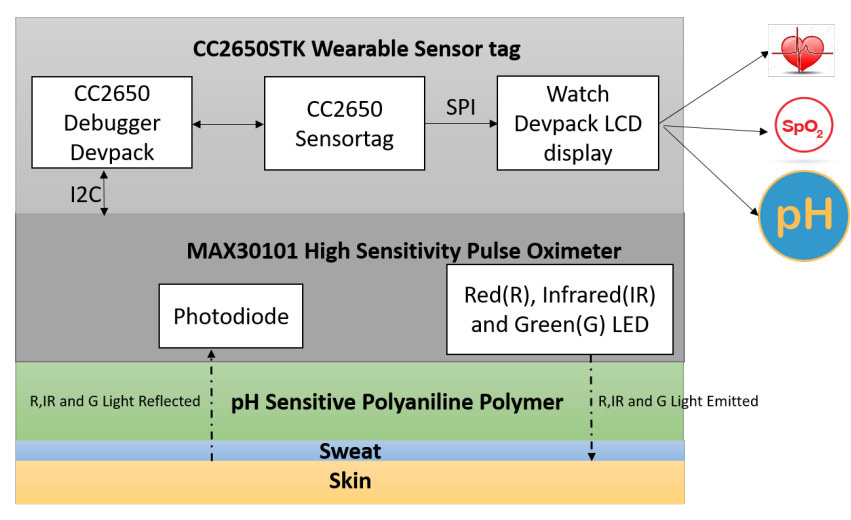

Figure 6: System level block diagram of our wearable prototype, pH-watch

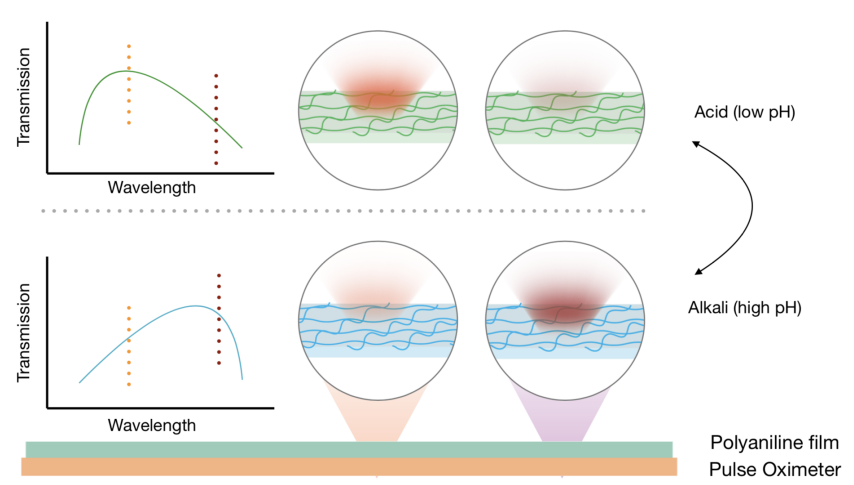

Figure 7: A schematic of a wearable PANI-based pH sensors combining with MAXIM pulse oximeter, which is an integrated light source and detector

protonation leads to a change in the optical property of the polymer, and results in strong transmission of light at $680 \mathrm{~nm}$ and strong absorbance of light at $880 \mathrm{~nm}$. When in contact with an alkaline (high $\mathrm{pH}$ ) solution, the polymer becomes deprotonated. In this state, the PANI absorbs strongly the light at $660 \mathrm{~nm}$ and transmits most of the light at $880 \mathrm{~nm}$. By measuring the relative transmission of light at these two wavelengths, the sensor can be used for real-time $\mathrm{pH}$ measurement of sweat.
3.2.2 Sensor fabrication. To improve skin contact and flexible conformity, we functionalized PANI on a supportive, flexible and transparent matrix prepared with polydimethylsiloxane (PDMS). The PDMS support was prepared by mixing the elastomer with the curing agent at a weight ratio of $10: 1$. The mixture was poured onto a master mould, and cured in an oven at $75{ }^{\circ} \mathrm{C}$ for $1 \mathrm{~h}$. Following curing, the PDMS layer is peeled from the master. The PDMS replica ( $2 \mathrm{~mm}$ height) was thoroughly washed with isopropanol. To functionalize the surface of PDMS with PANI film [26], we first exposed the PDMS surface to oxygen plasma for $60 \mathrm{~s}$ (Harrick Plasma). The activated PDMS was incubated with a $20 \%$ wt solution of $\mathrm{N}-[3$ (trimethoxylsilyl)propyl] aniline in ethanol for 60 mins [30]. Using this technique, a monolayer of silane-bearing aniline was formed on the substrate via molecular self-assembly. Chemical deposition of the PANI on the surface of PDMS was performed by immersing the PDMS with freshly prepared $1 \mathrm{M} \mathrm{HCl}$ solution containing the oxidant ( $0.25 \mathrm{M}$ ammonium peroxydisulfate) and $1 \mathrm{M}$ aniline. The pendant aniline on the surface served as the initiation site for polymerization and was also used to covalently anchor the PANI film on the substrate. The polymerization time was fixed to 1 day. After polymerization, the PDMS-PANI were washed extensively with water to remove any unattached PANI. The resulting films had good adhesion due to the chemical bonding between the substrate and polymer film.

The fabrication cost of our PANI-PDMS sensor is less than $\$ 1$ [31, 32]. Furthermore, owing to the process simplicity and the applicability for various shape and size casts, our technique allows for mass fabrication of our $\mathrm{pH}$ sensor [33].

3.2.3 Sensor performance measurements. We first evaluated the optical transmission spectra of the prepared PANI films using a plate reader. A plate reader is a device that emits light at specific wavelengths on the PANI film and measures the absorbance/ trasmission from the reflected light. Films were incubated in different $\mathrm{pH}$ solutions for $1 \mathrm{~min}$ before collection of the UV-Vis transmission spectra. As depicted in Figure 9(a), the PANI optical changes are highly sensitive to $\mathrm{pH}$ changes. As the $\mathrm{pH}$ was increased from 2 (acidic) to 12 (alkaline), the PANI film showed a shift in its absorption peak from $420 \mathrm{~nm}($ at $\mathrm{pH}$ 2) to $605 \mathrm{~nm}$ (at $\mathrm{pH} 12)$. This change is consistent with published studies on the different degree of protonation of the imine nitrogen atoms in the polymer chain [27]. We next plotted the $\mathrm{pH}$ dependence of the transmission 
(a)

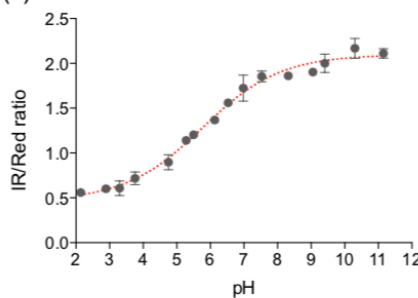

(b)

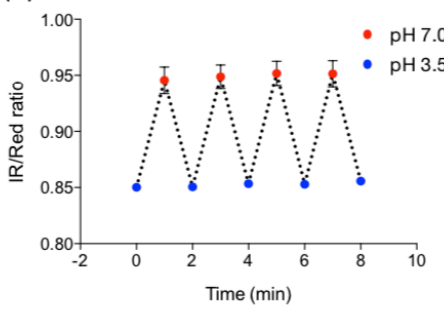

(c)

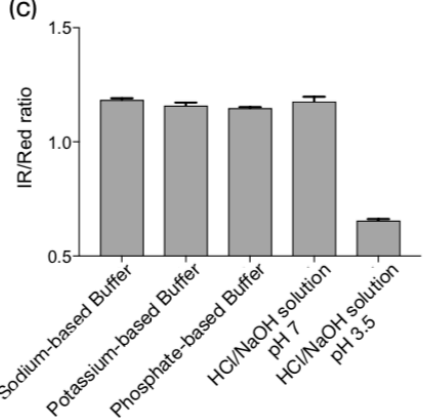

(d)

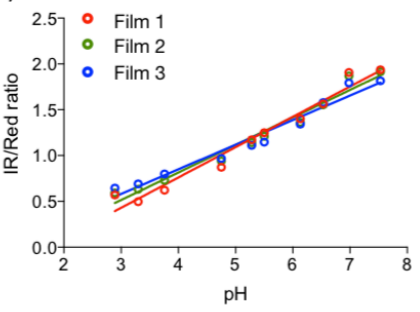

Figure 8: General performance of PANI film: (a) The ratio of the transmission intensity at $880 \mathrm{~nm}$ to the transmission intensity at $660 \mathrm{~nm}$, (b) repeatability, (c) selectivity, (d) reproducibility of PANI film
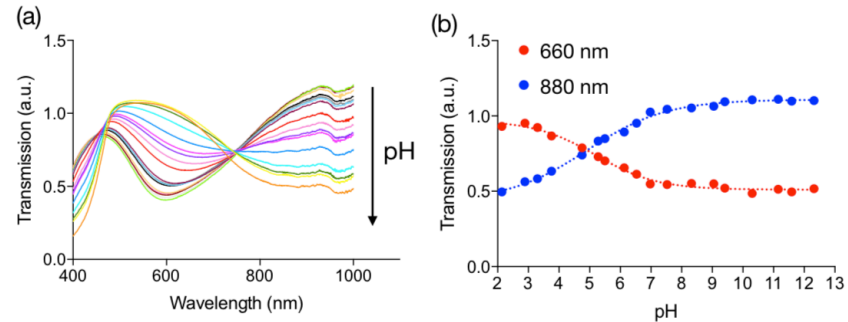

Figure 9: (a) Transmission spectra of the PANI coatings on PDMS when incubated in solutions at different $\mathrm{pH}(\mathrm{pH} 2-$ 12). (b) Transmission change of PANI film vs. $\mathrm{pH}$ at $660 \mathrm{~nm}$ and $880 \mathrm{~nm}$

at $660 \mathrm{~nm}$ and $880 \mathrm{~nm}$, respectively (Figure 9(b)). The characteristic PANI sigmoid shape curves were obtained with good correlations against a wide range of $\mathrm{pH}$ changes, $\mathrm{R}^{2}(660 \mathrm{~nm})=0.989$ and $\mathrm{R}^{2}(880 \mathrm{~nm})=0.996$. To further improve the detection accuracy and the material stability, we obtained the ratio of the transmission intensity at $880 \mathrm{~nm}$ to the transmission intensity at $660 \mathrm{~nm}$, as shown in Figure 8(a). As we varied the solution $\mathrm{pH}$ from 2 to 12 , this intensity ratio decreased and saturated. It is worth noting that this ratio change was most significant in the range of $\mathrm{pH} 3$ -8 , which is also the physiological $\mathrm{pH}$ fluctuation range of human sweat [34]. To demonstrate the repeatability of the sensor for measuring $\mathrm{pH}$ changes, we tested the integrated system repeatedly by using two different $\mathrm{pH}$ solutions, $\mathrm{pH} 3.4$ and $\mathrm{pH} 7.0$ prepared through mixing different amounts of $\mathrm{HCl}$ and $\mathrm{NaOH}$ solutions. As shown in Figure $8(\mathrm{~b})$, after changing the $\mathrm{pH}$ value of the solution alternately for 4 cycles, PANI film maintained a relatively consistent IR/Red ratio at the same $\mathrm{pH}$, indicating the repeatability of this material. The sensor not only demonstrated good reproducibility, to generate compatible signal outputs when treated with solutions of the same $\mathrm{pH}$, but also showed excellent responsiveness and repeatability. Because body sweat contains a variety of ions such as $\mathrm{Na}^{+}, \mathrm{K}^{+}, \mathrm{Cl}^{-}, \mathrm{P}^{5-}$ and $\mathrm{H}^{+}$, we next tested the specificity of the integrated sensor to $\mathrm{pH}$ changes amidst a complex ionic background. We chose four different solution mixtures, namely sodiumbased buffer, potassium-based buffer, phosphate-based buffer and
$\mathrm{HCl} / \mathrm{NaOH}$ solution ( $\mathrm{pH} 7$ ), respectively. As these solutions contain varying concentration of above mentioned ions $\left(\mathrm{Na}^{+}, \mathrm{K}^{+}, \mathrm{Cl}^{-}\right.$, $\mathrm{P}^{5-}$ and $\mathrm{H}^{+}$); these mixtures were prepared to $\mathrm{pH}=7$. Aside from these solutions, we also prepared a mixture which is $\mathrm{HCl} / \mathrm{NaOH}$ solution ( $\mathrm{pH}$ 3.5). We incubated the PANI film with these solutions for 1 minute and ratiometric measurements were performed. As demonstrated in Figure 8(c), the sensor is selectively responsive to $\mathrm{pH}$ changes. We finally tested the reproducibility of the film preparation in generating reliable analysis. We prepared multiple PANI films and measured the signal ratios of these sensors when incubated in various $\mathrm{pH}$ solutions. As shown in Figure 8(d), the sensors yielded highly uniform and robust signals, without demonstrating any significant deviations during the entire $\mathrm{pH}$ monitoring with a very small relative standard deviation (RSD) of $5.3 \%$.

\subsection{Calibration of $\mathrm{pH}$ versus $\mathrm{IR} / \mathrm{Red}$ ratio}

For sensing $\mathrm{pH}$ value from sweat, we need to first calibrate the reflected IR to Red ratio from MAX30101 mounted with PANI for different $\mathrm{pH}$ values. For calibrating IR/Red Ratio curve using MAX301-01, we created synthetic sweat solutions with $\mathrm{pH}$ value between 3 and 8 since $\mathrm{pH}$ of human sweat lies between 3 and 8 . We placed $200 \mu \mathrm{L}$ of each $\mathrm{pH}$ solution on MAX30101 and measured the average of the reflected IR to Red ratio for 30 seconds. We repeated the procedure for 3 times for each $\mathrm{pH}$ solution. For each $\mathrm{pH}$ value, we used the average of IR/Red ratios recorded during 3 different trials and calibrated the $\mathrm{pH}$ versus IR/Red ratio curve by performing a 4th order polynomial fit of the recorded IR/Red ratios for each $\mathrm{pH}$ solution. We used a 4 th order polynomial fit instead of linear fit because PANI does not produce IR/Red ratios in a linear trend as seen in Figure 8(a) and polynomial fit produces more accurate results than linear fit. Figure 10a shows the calibrated $\mathrm{pH}$ versus IR/Red ratio curve clearly showing the same increasing trends as the $\mathrm{pH}$ versus IR/Red ratio curve in Figure 8(a) obtained using plate reader. We also performed an ordinary least squares (OLS) fit between the IR/Red ratios obtained from plate reader and IR/Red ratios recorded from MAX30101. The resulting fit shown in Figure $10 \mathrm{~b}$ has a very high $R^{2}$ value of 0.981 indicating that the IR/Red ratios obtained from MAX30101 follow the same trend as the IR/Red ratios obtained using plate reader. 


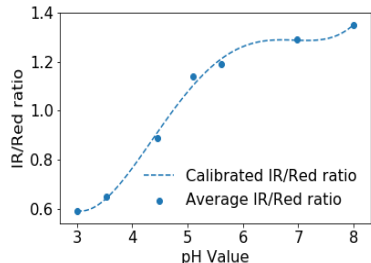

(a) IR/Red ratio curve calibration for $\mathrm{pH}$ range 3-8 using MAX30101

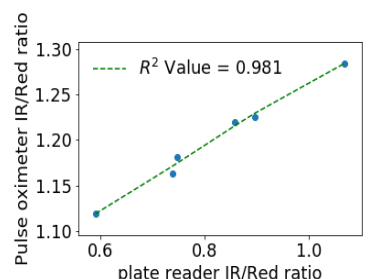

(b) $R^{2}$ plot between plate reader IR/Red ratio curve and MAX30101 IR/Red ratio curve
Figure 10: IR/Red ratio calibration for $\mathrm{pH}$ values

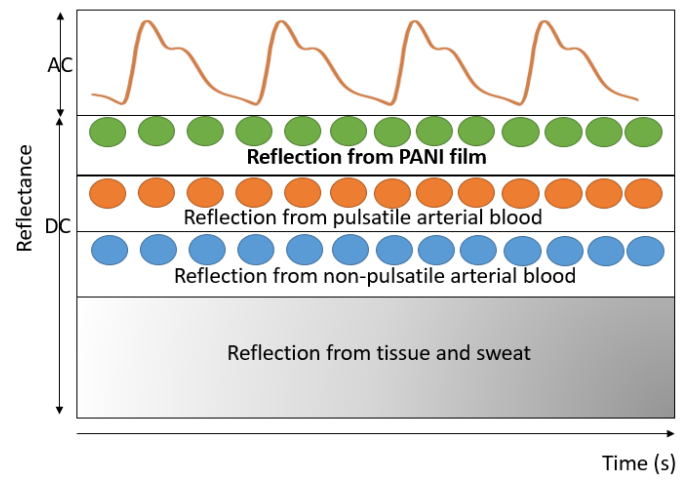

Figure 11: Components of pulse oximeter's reflection from skin

\subsection{Software algorithm for $\mathrm{pH}$ sensing}

When MAX30101 mounted with PANI is placed on the wrist with sweat, the reflected PPG signals comprise of 5 components as shown in Figure 11. The DC component of PPG signals come from four components - reflection from tissue and sweat, reflection from arterial non-pulsatile arterial blood, pulsatile arterial blood and reflection from PANI film. The AC component of the PPG signal comes from the pulsatile arterial blood. Therefore, reflected IR and Red components from the PANI film corresponding to $\mathrm{pH}$ value of sweat is present in the DC component of the signal. Also, a major portion of the DC component $(\approx 80 \%)$ of the PPG signal is contributed by the reflection from tissue. Therefore, we need to separate the DC component reflected from PANI from the overall DC component of the IR and Red PPG signals.

We define time series PPG signal recorded by $\mathrm{pH}$ Watch:

$$
I=[I(0), I(1), \ldots, I(M-1)], R=[R(0), R(1), \ldots, R(M-1)]
$$

where $I$ and $\mathrm{R}$ are the reflected infrared and red PPG signals respectively recorded by $\mathrm{pH}$ Watch mounted with PANI. $\mathrm{M}$ is the number of samples. $\mathrm{pH}$ Watch operates at a sampling frequency $f_{s}=100 \mathrm{~Hz}$, sample averaged by 4 since MAX30101 chip can send data by averaging adjacent samples to reduce throughput and we calculate $\mathrm{pH}$ for every sliding time window of $\mathrm{T}=5$ seconds with overlap of $\mathrm{S}=3$ seconds So, $M=f_{S} T=125$ samples. $D C_{I}$ and $D C_{R}$ are the DC components of infrared and red PPG signal respectively. Infrared and red LEDs are switched on alternatively in MAX30101 to avoid self heating and reduce power consumption. Although IR and Red pulse repetition frequency can go up to $100 \mathrm{KHz}$, we chose $100 \mathrm{~Hz}$ as the sampling rate since low sampling frequency will also reduce power consumption.

Our goal is to find the DC component reflected from PANI that corresponds to the $\mathrm{pH}$ value of sweat given by $D C_{I}^{P A N I}$ and $D C_{R}^{P A N I}$ from $D C_{I}$ and $D C_{R}$ respectively. $D C_{I}$ and $D C_{R}$ are calculated by finding the mean of I and R PPG signals recorded by $\mathrm{pH}$ Watch respectively given by,

$$
D C_{I}=\frac{1}{M} \sum_{n=0}^{M-1} I(n) \quad \text { and } \quad D C_{R}=\frac{1}{M} \sum_{n=0}^{M-1} R(n)
$$

In order to find $D C^{P A N I}$ from the DC component of the PPG signal, we need to first separate the reflected DC component from tissue and pulsatile as well as non-pulsatile arterial blood denoted as $D C^{\overline{P A N I}}$ which cannot be calculated deterministically. However, the DC components reflected from the skin, tissue, fat, bones etc. remain constant over time. Also, the blood volume in the veins and capillaries remains constant and thus, reflected DC components of PPG signals are constant over time [35]. So, before starting $\mathrm{pH}$ measurements, we initialize the $D C^{\overline{P A N I}}$ as the average of DC components measured from the wrist without sweat for 3 seconds. At the start of real-time $\mathrm{pH}$ sensing, since there will not be any sweat on the skin for PANI sensor to react with, we will not consider the $\mathrm{pH}$ of skin. This average of reflected DC components measured from wrist devoid of sweat for 3 seconds is denoted by $D C_{I}^{\text {init }}$ and $D C_{R}^{\text {init }}$ for infrared and red respectively. Using the initialized values, we can find $D C^{P A N I}$ by subtracting $D C^{\text {init }}$ from the $D C$ component of the PPG signal for each time window. Therefore, we have

$$
D C_{I}^{P A N I}=D C_{I}-D C_{I}^{i n i t}, D C_{R}^{P A N I}=D C_{R}-D C_{R}^{i n i t}
$$

From this, IR/Red ratio can be calculated as:

$$
I R / \text { Red ratio }=\frac{D C_{I}^{P A N I}}{D C_{R}^{P A N I}}
$$

Using the calculated IR/Red ratio, we can use the $\mathrm{pH}$ versus IR/Red ratio calibration obtained in Figure 10a and calculate the $\mathrm{pH}$ of sweat for every time window of 5 seconds. Our $\mathrm{pH}$ estimation approach does not suffer from motion artifacts caused by hand swing since typical frequency of motion artifacts is in between $0.5 \mathrm{~Hz}$ $5 \mathrm{~Hz}$ [36]. We evaluated our $\mathrm{pH}$ estimation approach in $\mathrm{pH}$ Watch with on-body trials on 10 participants in Section 4.3, showing an accuracy of $\approx 91 \%$ in estimating $\mathrm{pH}$ with error of $\pm 0.6 \mathrm{pH}$ in realtime.

\subsection{Software algorithm for heart rate sensing}

3.5.1 HR estimation using TROIKA framework. Pulse oximeters shine red, infrared and green light on the skin and measure heart rate (HR) from the reflected green photoplethysmograph (PPG) signals. For heart rate measurements, we use green PPG signals instead of red or IR PPG signals because the wrist has less blood perfusion and a higher energy PPG signal (green light) is preferable. The MAX30101 has three LEDs namely red, infrared and green. As explained earlier, we use the IR and red PPG signals 


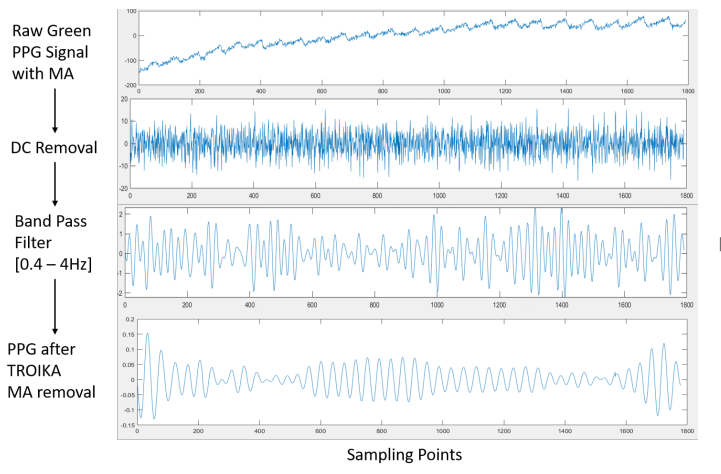

Figure 12: Illustration of motion artifact removal using signal decomposition

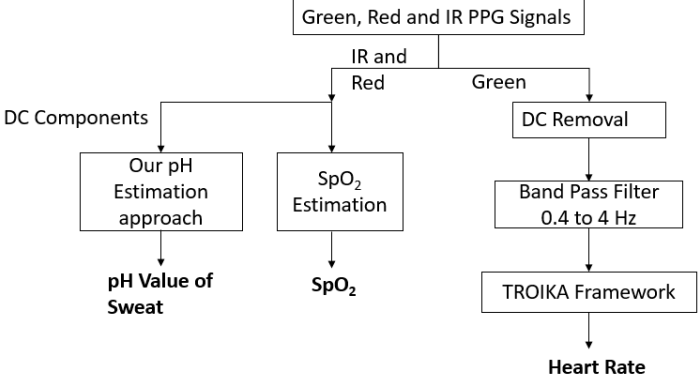

Figure 13: Software flow in TI sensor tag

\begin{tabular}{l|l|l|}
\hline $\begin{array}{l}\text { pH } \\
\text { value } \\
\text { of the } \\
\text { Solution }\end{array}$ & $\begin{array}{l}\text { Esti- } \\
\text { mated } \\
\text { pH } \\
\text { Value }\end{array}$ & $\begin{array}{l}\% \\
\text { error }\end{array}$ \\
\hline 3.54 & 3.45 & 2.5 \\
\hline 4.46 & 4.57 & 2.5 \\
\hline 4.98 & 4.87 & 2.2 \\
\hline 5.5 & 5.63 & 2.4 \\
\hline 6.13 & 6.22 & 1.8 \\
\hline 6.98 & 6.84 & 2.0 \\
\hline 7.54 & 7.43 & 1.5 \\
\hline Average error (\%) & $2.13 \%$ \\
\hline
\end{tabular}
Table 2: pH of syn-
thetic solutions
measured by commer-
cial pH meter and
pH-Watch

for sweat $\mathrm{pH}$ estimation. For heart rate monitoring, in addition to the IR and red PPG signals, we also use the green PPG signals.

PPG signals are greatly affected by motion artifacts (MA) which interfere with the measurement of heart rate. Acceleration data has been shown to be an effective solution for removing MA from PPG signals even during intense physical exercise. The CC2650 sensor tag is equipped with accelerometer, so we made use of both the accelerometer readings from CC2650 sensor tag and the green PPG signals obtained from MAX30101 to remove MA from PPG signal using the light-weight TROIKA framework [8]. TROIKA consists of three main parts - (a) Signal decomposition (b) Sparse Signal Reconstruction and (c) Spectral Peak tracing.

In TROIKA framework, PPG signals are processed in sliding time windows of $\mathrm{T}$ seconds with incremental steps of $\mathrm{S}$ seconds preferably $S \leq T / 2$ and $\mathrm{HR}$ is estimated at each time window of $\mathrm{T}$ seconds. In $\mathrm{pH}$ Watch, We chose $\mathrm{T}=5$ seconds and $\mathrm{S}=2$ seconds. Before feeding the PPG signals into the TROIKA framework, the following preprocessing is done on the signal:

(1) DC removal is performed on the PPG Signal to achieve a baseline detrended signal with zero mean

(2) The PPG signal and 3-axis accelerometer data are band pass filtered between 0.4 and $5 \mathrm{~Hz}$ to remove noise and MA lying outside the heart rate frequency.

Figure 12 shows a cleansed PPG signal obtained after preprocessing and TROIKA MA removal. Although we use TROIKA [8] in $\mathrm{pH}$ Watch, our primary aim is to demonstrate that the addition of PANI film to the pulse oximeter does not hinder the ability of pulse oximeter in measuring heart rate even during motion. Therefore, when PANI is mounted on current day fitness trackers, any custom motion artifact removal algorithm developed by the manufacturers of the fitness trackers can also be used to estimate the heart rate even during intense physical exercise.

3.5.2 $\mathrm{SpO}_{2}$ estimation. For estimating $\mathrm{SpO}_{2}$, we need to calculate the ratio R presented in Equation 1 from the $\mathrm{AC}$ and DC components of the Red and Infrared signals respectively. In order to handle motion artifacts, we use the cleansed PPG signal obtained using Singular spectral analysis (SSA) [8] as the AC component of the IR and red PPG signals. For $\mathrm{SpO}_{2}$ measurements, we calculate the ratio $\mathrm{R}$ between infrared and red PPG signals as defined in Equation 1. The scattering constant and the optical path length vary considerably between the red and infrared wavelengths. Thus, the calculated ratio $\mathrm{R}$ cannot be directly related to the physiological oxygen saturation $\left(\mathrm{SpO}_{2}\right)$ [37]. Therefore the relationship between ratio $\mathrm{R}$ and the physiological $\mathrm{SpO}_{2}$ for any commercial pulse oximeter is determined experimentally through calibration. For any pulse oximeter, $\mathrm{R}$ is determined for many healthy volunteers using the pulse oximeters and their $\mathrm{SpO}_{2}$ is measured by collecting their arterial blood. Using the obtained $\mathrm{R}$ and $\mathrm{SpO}_{2}$ values, a curvilinear approximation or empirical calibration of pulse oximeter is made to obtain a more precise $\mathrm{SpO}_{2}$ measurement. In $\mathrm{pH}$ Watch, the standard calibration equation recommended by MAX30101 $S p O_{2}=104-17 R$ is used to estimate the $\mathrm{SpO}_{2}$ value.

\subsection{Software stack of $\mathrm{pH}-$ Watch}

In $\mathrm{pH}$-Watch, all the software algorithms for $\mathrm{pH}$, heart rate and $\mathrm{SpO}_{2}$ estimation are performed on the $\mathrm{CC} 2650$ sensortag for every time window of 5 seconds. The number of samples for processing is constrained at $M=F_{S} T / 4=100 \times 5 / 4=125$ samples due to the small SRAM in CC2650. However, we can also process more samples with a faster $\mathrm{CPU}$ and increase the time window $\mathrm{T}$ of processing. The software flow of $\mathrm{pH}$ Watch shown in Figure 13 is as follows:

(1) MAX30101 shines red, infrared and green light on the wrist with sweat and reads the reflected PPG signals and sends the ADC values of the PPG signals over I2C bus to CC2650 for 2 seconds. Accelerometer readings from CC2650 are also read for the same 2 seconds. The samples are read and stored in $6 \mathrm{~KB}$ sensor controller in CC2650.

(2) Green PPG Signals are preprocessed with DC removal and band pass filtering. Accelerometer readings are also band pass filtered. Then, heart rate is calculated using TROIKA framework for motion artifact removal.

(3) Infrared and red PPG signals are read, DC and AC components extracted and $\mathrm{SpO}_{2}$ values calculated.

(4) Finally, DC components of IR and red PPG signals are used to calculate the $\mathrm{pH}$ value of sweat using our $\mathrm{pH}$ estimation approach.

(5) All the calculated $\mathrm{pH}$ value, Heart rate and $\mathrm{SpO}_{2}$ values are shown on Watch Devpack LCD display (see Figure 5(d)). 
(6) Repeat step(1).

First two seconds of PPG signals and intermediate values are flushed immediately after computation. Our source code in CC2650 spans $\approx 800$ lines and takes $\approx 4$ seconds for execution.

In $\mathrm{pH}$ Watch, before beginning to exercise or be involved in any activity that stimulates sweating, we require the user to press the user button in CC2650 which records the average of DC components of infrared and red PPG signals reflected from wrist without sweat.

\section{EXPERIMENTAL EVALUATION}

\subsection{Accuracy of $\mathrm{pH}$-Watch on synthetic $\mathrm{pH}$ solutions}

Firstly, we evaluated MAX30101 mounted with PANI without placing it on skin to compute the accuracy of PANI in estimating $\mathrm{pH}$ using calibration curve in Figure 10a. We created synthetic buffer solutions of different $\mathrm{pH}$ value by mixing different concentrations of $\mathrm{Hcl}$ and $\mathrm{NaOH}$. We used commercial wireless $\mathrm{pH}$ meter "HI14142 - HALO® Wireless pH Meter" from Hannah Instruments for measuring the $\mathrm{pH}$ value of each solution. HI14142 is also compatible with measuring $\mathrm{pH}$ value of sweat directly from the skin. We placed $200 \mu \mathrm{L}$ of each solution on PANI and measured the $\mathrm{pH}$ values from the reflected IR/Red ratios. Table 2 shows $\mathrm{pH}$ value of different solutions measured by commercial $\mathrm{pH}$ meter and $\mathrm{pH}$ value measured by MAX30101 mounted with PANI. The average error between the $\mathrm{pH}$ measured by commercial $\mathrm{pH}$ meter and estimated $\mathrm{pH}$ value was found to be $2.13 \%$. We can see that the estimated $\mathrm{pH}$ value varies from the commercial $\mathrm{pH}$ meter readings by at most $2.5 \%$ which is comparable to $<2.2 \% \mathrm{pH}$ variations in PANI based electrochemical $\mathrm{pH}$ sensor reported in [15].

\subsection{Accuracy of $\mathrm{pH}-$ Watch in heart rate (HR) and $\mathrm{SpO}_{2}$ measurements}

We evaluated the accuracy of $\mathrm{pH}$ watch in heart rate and $\mathrm{SpO}_{2}$ measurements with and without motion artifacts. We recruited a participant who wore the $\mathrm{pH}$ watch on his left wrist and MAX30102 high sensitivity finger pulse oximeter (with IR and Red LED) on his right hand serving as a ground truth for HR and $\mathrm{SpO}_{2}$ measurements. The measurements from MAX30102 were measured and logged using the MAX30102ACCEVKIT evaluation board which reads in the finger PPG measurements and calculates $\mathrm{HR}$ and $\mathrm{SpO}_{2}$. During the experiment, we restrained the participant from not moving his right hand so that the ground truth HR and $\mathrm{SpO}_{2}$ values are not affected by MA. Each experiment was carried out for 120 seconds. The experiment was repeated 8 times without motion in the left hand with $\mathrm{pH}$-Watch for testing accuracy without motion and 8 times with participant being asked to induce random motion in left hand continuously by hand swings and heavy to and fro shaking of left hand, for testing accuracy in the presence of MA. For experiments with motion in left hand, we asked the user to stay stationary for first 3 seconds, since TROIKA requires initialization of heart rate. Finally, for each experiment we compared the resulting $\mathrm{HR}$ and $\mathrm{SpO}_{2}$ measurements of $\mathrm{pH}-$ Watch against the ground truth $\mathrm{HR}$ and $\mathrm{SpO}_{2}$ measurements recorded by finger pulse oximeter.
4.2.1 Accuracy of $\mathrm{HR}$ and $\mathrm{SpO}_{2}$ measurements without motion artifacts. Table 3 shows HR measurements recorded by $\mathrm{pH}$ Watch without hand motion validated against finger pulse oximeter readings which serves as ground truth. Average percentage error of $\mathrm{HR}$ and $\mathrm{SpO}_{2}$ measurements were $1.22 \%$ and $2.75 \%$ respectively with $\mathrm{HR}$ and $\mathrm{SpO}_{2}$ varying by a maximum of $<3.44 \%$ and $<3.22 \%$ respectively. The errors observed were negligible and may be attributed to the fact that PPG readings from finger are inherently more accurate than PPG readings from wrist due to the better blood perfusion in fingers.

4.2.2 Accuracy of $\mathrm{HR}$ and $\mathrm{SpO}_{2}$ measurements with motion artifacts. Table 4 shows HR measurements recorded by $\mathrm{pH}$ Watch with random hand motions validated against finger pulse oximeter readings serving as ground truth. Average percentage error of HR and $\mathrm{SpO}_{2}$ measurements were $4.98 \%$ and $4.57 \%$ respectively with $\mathrm{HR}$ and $\mathrm{SpO}_{2}$ varying by a maximum of $<6.41 \%$ and $<6.73 \%$ respectively. This is comparable to the average heart rate error of $1.8 \%$ with maximum variation of $4.70 \%$ as reported originally with TROIKA [8]. The small difference in error rate is due to the fact that $\mathrm{pH}$ Watch uses very small time windows of 5 seconds owing to RAM limitation in CC2650. [8] used time windows of $10 \mathrm{sec}-$ onds with 1250 samples and hence can have more accurate HR measurements than $\mathrm{pH}$ Watch. The average errors could be further reduced with a more powerful CPU with higher RAM, which most current day wearables already have. For instance, the latest Samsung Galaxy watch has a $1.15 \mathrm{GHz}$ Exynos 9110 processor with 768MB RAM. Thus, the experiments clearly show that $\mathrm{pH}$ Watch with PANI does not hinder the normal ability of pulse oximeters in measuring HR and blood oxygen concentration, thereby confirming that our $\mathrm{pH}$ sensing approach could be easily integrated with all current-day smart watches or fitness trackers with the addition of our PANI sensor.

\subsection{On-body trials}

To evaluate the accuracy and efficiency of $\mathrm{pH}$ watch in real-time monitoring of $\mathrm{pH}$ from human sweat, we recruited 10 participants and conducted on-body-trials with $\mathrm{pH}$ Watch for measuring their sweat $\mathrm{pH}$ value.

For the first experiment to test the accuracy of $\mathrm{pH}$ Watch in sensing $\mathrm{pH}$ value from sweat, we recruited 6 participants. Each participant was given a randomized ID and the data collected from them where mapped to the randomized ID to protect their personal data. Each participant was asked to wear $\mathrm{pH}$ Watch and exercise for 10-20 minutes so that they start perspiring. Before beginning to exercise, the tissue DC of the participants given by $D C^{\text {init }}$ were measured for 3 seconds by pressing the user button in $\mathrm{pH}$ Watch. Once the participants started to sweat, we compared the $\mathrm{pH}$ value of sweat measured by $\mathrm{pH}$ watch against the $\mathrm{pH}$ value of sweat by means of a HI14142 wireless $\mathrm{pH}$ meter to calculate the accuracy. The above procedure is repeated twice for each participant.

In the second experiment to assess the capability of $\mathrm{pH}$ Watch for real-time continuous sensing of $\mathrm{pH}$ value and detection of dehydration during exercise, we recruited 4 participants who wore $\mathrm{pH}-W a t c h$ and cycled in the gym for 80 minutes. The $\mathrm{HR}, \mathrm{SpO}_{2}$ 


\begin{tabular}{|c|c|c|c|c|c|c|}
\hline \multirow[t]{2}{*}{ Data } & \multicolumn{2}{|l|}{$\begin{array}{l}\text { HR } \\
\text { (BPM) }\end{array}$} & \multirow{2}{*}{$\begin{array}{l}\% \\
\text { Error }\end{array}$} & \multicolumn{2}{|l|}{$\begin{array}{l}\mathrm{SpO}_{2} \\
(\%)\end{array}$} & \multirow{2}{*}{$\begin{array}{l}\% \\
\text { Error }\end{array}$} \\
\hline & $\begin{array}{l}\text { pH } \\
\text { Watch }\end{array}$ & $\begin{array}{l}\text { MAX } \\
30102\end{array}$ & & $\begin{array}{l}\text { pH } \\
\text { Watch }\end{array}$ & $\begin{array}{l}\text { MAX } \\
30102\end{array}$ & \\
\hline 1 & 85 & 86 & 1.16 & 92.5 & 95 & 2.63 \\
\hline 2 & 82 & 84 & 2.38 & 91 & 94 & 3.2 \\
\hline 3 & 85 & 83 & 2.35 & 94 & 97 & 3.1 \\
\hline 4 & 83 & 82 & 1.2 & 96 & 98 & 2.04 \\
\hline 5 & 74 & 75 & 1.33 & 93 & 95.5 & 2.61 \\
\hline 6 & 83 & 84 & 1.19 & 94 & 96 & 2.1 \\
\hline 7 & 84 & 87 & 3.44 & 95 & 98 & 3.06 \\
\hline \multirow[t]{2}{*}{8} & 73 & 73 & 0 & 91 & 93 & 3.22 \\
\hline & \multicolumn{2}{|c|}{$\begin{array}{l}\text { Average HR } \\
\text { \% Error }\end{array}$} & 1.22 & \multicolumn{2}{|c|}{$\begin{array}{l}\text { Average } \mathrm{SpO}_{2} \\
\text { \% Error }\end{array}$} & 2.75 \\
\hline
\end{tabular}

Table 3: HR and $\mathrm{SpO}_{2}$ measurements by $\mathrm{pH}-$ Watch and MAX30102 in the absence of motion

\begin{tabular}{|c|c|c|c|c|c|c|}
\hline \multirow[t]{2}{*}{ Data } & \multicolumn{2}{|l|}{$\begin{array}{l}\text { HR } \\
\text { (BPM) }\end{array}$} & \multirow{2}{*}{$\begin{array}{l}\% \\
\text { Error }\end{array}$} & \multicolumn{2}{|l|}{$\begin{array}{l}\mathrm{SpO}_{2} \\
(\%)\end{array}$} & \multirow{2}{*}{$\begin{array}{l}\% \\
\text { Error }\end{array}$} \\
\hline & \begin{tabular}{l|} 
pH \\
Watch
\end{tabular} & $\begin{array}{l}\text { MAX } \\
30102\end{array}$ & & $\begin{array}{l}\text { pH } \\
\text { Watch }\end{array}$ & $\begin{array}{l}\text { MAX } \\
30102\end{array}$ & \\
\hline 1 & \begin{tabular}{|l|}
83 \\
\end{tabular} & 88 & 5.68 & \begin{tabular}{|l|}
93.5 \\
\end{tabular} & 98 & 4.59 \\
\hline 2 & 82 & 78 & 4.87 & 92 & 97 & 5.15 \\
\hline 3 & 81 & 86 & 5.8 & 93 & 96 & $\begin{array}{ll}3.2 \\
\end{array}$ \\
\hline 4 & 80 & 76 & 5.26 & 89 & 94 & 5.32 \\
\hline 5 & 79 & 75 & 5.33 & 91 & 95 & 4.21 \\
\hline 6 & 78 & 81 & 3.7 & 90 & 96.5 & 6.73 \\
\hline 7 & 70 & 72 & 2.78 & 92 & 94.5 & 2.12 \\
\hline \multirow[t]{2}{*}{8} & \begin{tabular}{|l|}
73 \\
\end{tabular} & 78 & 6.41 & 90 & 95 & 5.26 \\
\hline & \multicolumn{2}{|c|}{$\begin{array}{l}\text { Average HR } \\
\text { \% Error }\end{array}$} & 4.98 & \multicolumn{2}{|c|}{$\begin{array}{l}\text { Average } \mathrm{SpO}_{2} \\
\% \text { Error }\end{array}$} & 4.57 \\
\hline
\end{tabular}

Table 4: $\mathrm{HR}$ and $\mathrm{SpO}_{2}$ measurements by $\mathrm{pH}^{-}$ Watch and MAX30102 in the presence of MA and $\mathrm{pH}$ values measured by $\mathrm{pH}$ watch were logged. During exercise, we measure the ground truth $\mathrm{pH}$ value manually using wireless $\mathrm{pH}$ meter every 5 or $10 \mathrm{mins}$ due to the limited output rate of our wireless $\mathrm{pH}$ meter. Our wireless $\mathrm{pH}$ meter takes around $30 \mathrm{sec}-$ onds to give a stable reading. This does not affect our evaluations because $\mathrm{pH}$ of sweat changes very slowly with exercise and does not change considerably within 10 mins.

Our evaluations took into consideration a variety of skin tones, from Chinese skin types to moderately dark skinned Indian people between 21-30 years of age (Chinese, Malays and Indians). All our participants were males. A summary of skin types and demographics of the participants is shown in Table 6 .

4.3.1 Accuracy of $\mathbf{p H}$ Watch. Table 7 shows $\mathrm{pH}$ measurements made by $\mathrm{pH}$ watch validated against the $\mathrm{pH}$ value of sweat measured by wireless $\mathrm{pH}$ meter during on-body trials by following the first experimental procedure. Average percentage error was $2.31 \%$ from the $\mathrm{pH}$ meter readings with the maximum variation of $<4.3 \%$. We also plotted the $\mathrm{pH}$ versus IR/Red ratio curve for $\mathrm{pH}$ values we measured during this experiment as shown in Figure 14 and the curve resembles the calibration curve in Figure 10a. We observed a $R^{2}$ value of 0.99 indicating very high correlation between the two curves. This is similar to previous wearable $\mathrm{pH}$ sensing approaches showing - (1) maximum variation of $<2.2 \%$ reported by PANI based electrochemical $\mathrm{pH}$ sensing approach [15] and (2) maximum variation of $<8.3 \%$ ( $\pm 0.5 \mathrm{pH}$ change) reported by ORMOSIL textile fabric based optical $\mathrm{pH}$ sensing approach [12]. This indicates that $\mathrm{pH}$ Watch has sensing accuracy comparable with current state-of-art $\mathrm{pH}$ sensors. Yet, it is long-term reusable and compatible with today's pervasive wearables.

4.3.2 Real-time continuous monitoring of $\mathrm{HR}, \mathrm{SpO}_{2}$ and pH. Figure 15 shows $\mathrm{HR}, \mathrm{SpO}_{2}$ and $\mathrm{pH}$ measurements of a participant continuously recorded by $\mathrm{pH}$ watch during the second experimental procedure for assessing the real-time sensing capacity of $\mathrm{pH}$ Watch. The real-time heart rate measurements show that:

(1) In the beginning of the exercise, the heart rate spiked from 70 to 90 BPM within the first 5 minutes indicating that the exercise had started.

(2) HR remained almost constant for the next 10 minutes around $90 \mathrm{BPM}$ and again increased to $100 \mathrm{BPM}$ indicating that the exercise is picking up and the participant is cycling faster now.
(3) HR remained around 110 BPM for the next 15 minutes and then increased to $120 \mathrm{BPM}$ and stabilized around 120BPM. This increase can be due to decrease in hydration levels in human body as perspiration happens during exercise. This drop in hydration levels of human body causes mild strain on the heart since blood volume decreases. Also blood will be carrying more sodium as a result of decreased hydration levels which makes it more difficult for the heart to pump blood. Therefore the heart beats faster resulting in increased HR.

On the other hand, real-time $\mathrm{SpO}_{2}$ measurements remain consistent, around $97 \%$ most of the time with minimum $\mathrm{SpO}_{2}$ around $96 \%$ and maximum $\mathrm{SpO}_{2}$ around $98 \%$. This agrees well with the fact that $\mathrm{SpO}_{2}$ levels would regulate themselves and remain stable during low and middle intensity exercise where the person breathes in enough oxygen continuously during exercise.

Figure 15 shows real time $\mathrm{pH}$ values validated against $\mathrm{pH}$ values of sweat measured by the wireless $\mathrm{pH}$ meter. Real time $\mathrm{pH}$ values increase with time during exercise. For the first 20 minutes, the participant did not sweat and hence no $\mathrm{pH}$ readings were shown in the graph. It took 20 minutes for the participant to sweat because the wrist region does not sweat so fast. After 20 mins, the participant started to sweat and for the next 20 mins, the $\mathrm{pH}$ value of the sweat remained stable around 5.2 to 5.3. This stability in $\mathrm{pH}$ value of sweat is because the sweat rate will be very limited during the moderate phase of exercising. Once the user starts to kick in with increasing the intensity of workout, the $\mathrm{pH}$ value of sweat rises from 5.3 to 5.7 denoting increase in the sweat rate of the participant. This is also appropriately indicated in the heart rate measurements where there is a clear increase in the heart rate to 120 BPM due to increase in sweat rate and reduction in hydration levels. Then the $\mathrm{pH}$ slowly increased from 5.7 to 5.8 for the next 20 minutes and again increased to 6.2 within 10 minutes. This also confirms that, as the person exercises for a prolonged duration (7080 minutes), his sweat rate increases and also the concentration of sodium in his sweat increases. This in turn, increases the $\mathrm{pH}$ value of sweat.

We repeated the same experimental procedure with 3 other participants and the real-time $\mathrm{pH}, \mathrm{HR}$ and $\mathrm{SpO}_{2}$ values measured by $\mathrm{pH}$ Watch is shown in Figure 15. Figure 16 shows the cumulative distribution function of $\mathrm{pH}$ errors for each of the 4 participants. 


\begin{tabular}{|l|l|l|l|l|}
\hline $\begin{array}{l}\text { Skin } \\
\text { Type }\end{array}$ & $\begin{array}{l}\text { Skin } \\
\text { Color }\end{array}$ & $\begin{array}{l}\text { Number of } \\
\text { participants }\end{array}$ & $\begin{array}{l}\text { Age } \\
\text { anger of } \\
\text { participants } \\
\text { who took part } \\
\text { in continuous } \\
\text { sensing }\end{array}$ \\
\hline $\begin{array}{l}\text { Chinese } \\
\text { /Malay }\end{array}$ & $\begin{array}{l}\text { Light- } \\
\text { skinned }\end{array}$ & 5 & $21-30$ & 2 \\
\hline Indian & $\begin{array}{l}\text { Light- } \\
\text { brown }\end{array}$ & 3 & $24-27$ & 2 \\
\hline Indian & $\begin{array}{l}\text { Dark- } \\
\text { brown }\end{array}$ & 2 & $24-27$ & 0 \\
\hline
\end{tabular}

Table 6: Skin characteristics of participants in trials

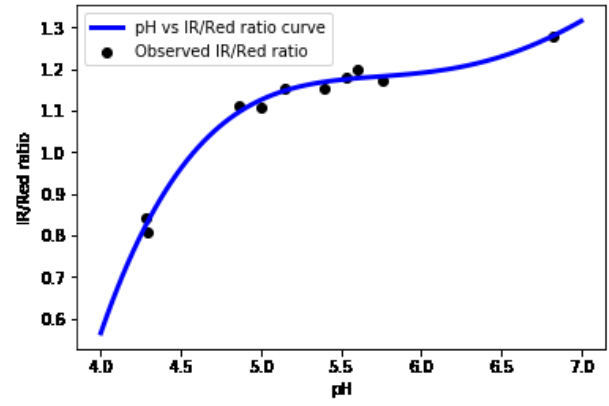

Figure 14: $\mathrm{pH}$ versus IR/red ratio curve for on-body trials

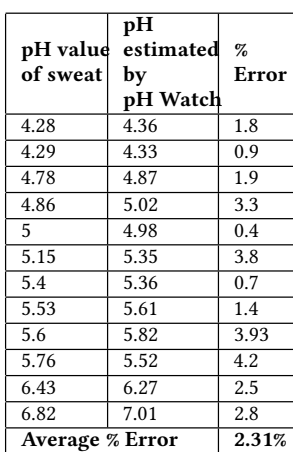

Table 7: $\mathrm{pH}$ measured by $\mathrm{pH}$ Watch during on-body trials
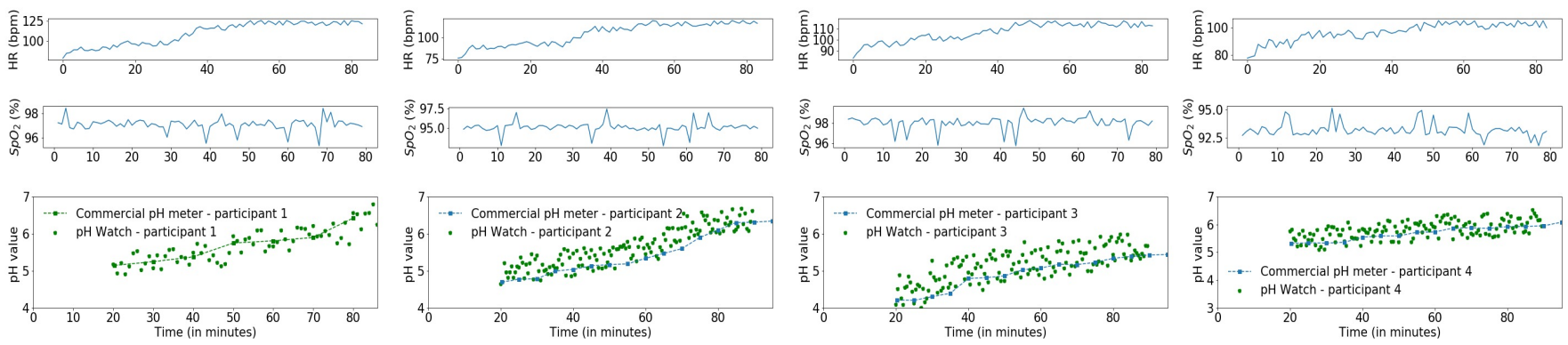

Figure 15: Real-time continuous monitoring of $\mathrm{pH}$, heart rate and $\mathrm{SpO}_{2}$ of 4 participants

The median $\mathrm{pH}$ error for all the participants ranged from 0.2 to 0.28 . The maximum $\mathrm{pH}$ error ranged from 0.4 to 0.6 . Therefore, $\mathrm{pH}$ values measured by $\mathrm{pH}$ Watch differed from the wireless $\mathrm{pH}$ meter by a maximum of $\pm 0.6 \mathrm{pH}$ change comparable to the results reported in [12] and also shows that $\mathrm{pH}$ Watch can detect $\mathrm{pH}$ with an accuracy of $\approx 91 \%$. This clearly indicates the potential of $\mathrm{pH}$ Watch in detecting dehydration levels in human body during exercise and future research on correlation between heart rate and $\mathrm{pH}$ value of sweat could prove to be helpful in detecting an accurate dehydration risk with just the addition of an low cost off-the-shelf $\mathrm{pH}$ sensitive PANI to the pulse oximeters being used in current day fitness trackers.

For our real-time continuous sensing experiment, we had incorporated participants of Chinese skin type and Indian skin type. Figure 17 shows $\mathrm{pH}$ errors reported by Indian skin and Chinese skin types. We can observe that Indian skin and Chinese skin had similar average $\mathrm{pH}$ errors of 0.2 and 0.27 respectively which validates that our $\mathrm{pH}$ measurement shows similar errors for different skin types and remain relatively unaffected by skin color. More intuitively, skin colour has little effect on our $\mathrm{pH}$ measurements, because skin colour contributes to the DC part of the PPG signal which is measured during the initialization part of our $\mathrm{pH}$ sensing algorithm and removed during the $\mathrm{pH}$ measurement.

\subsection{Power consumption of $\mathrm{pH}$ Watch}

The entire $\mathrm{pH}$-watch prototype consists of electronic components (MAX30101, TI sensor tag) and the passive component, our PANIbased flexible sensor which does not require power. We measured

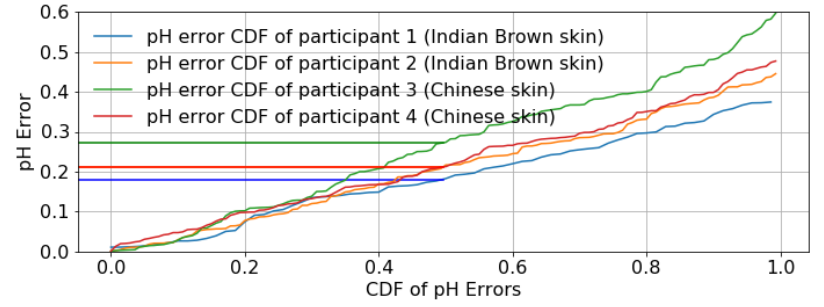

Figure 16: Cumulative distribution function of $\mathrm{pH}$ errors of all 4 participants
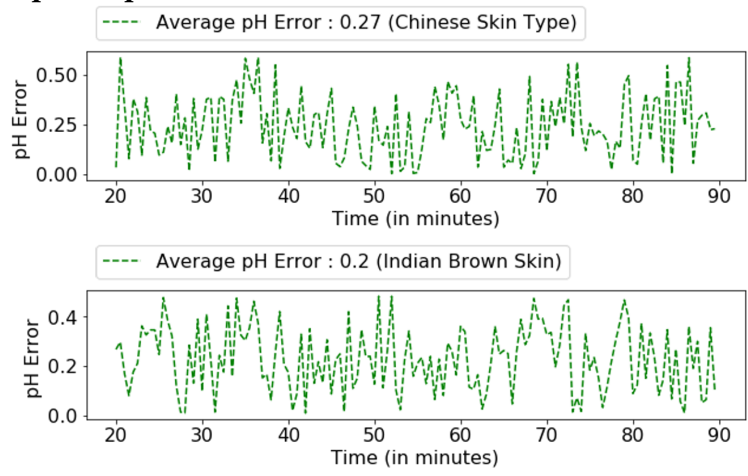

Figure 17: pH errors of Indian and Chinese skin types

the power dissipation of the full system when the pulse oximeter chip is sending encoded results to CC2650 chipset and the chipset 
is running algorithms for $\mathrm{pH}$ value, $\mathrm{SpO}_{2}$ and heart rate, with the debugger devpack and display. Power measurement is done with the Monsoon FTA22D power monitor. The MAX30101 and sensortag are powered by a single supply, the $5 \mathrm{~V}$ voltage output from the monitor using a USB interface with two split ports.

For a 10-second measurement, the power drawn from the system averaged $487 \mathrm{~mW}$ whereas the pulse oximeter consumes 139.4 $\mathrm{mW}$ on average. As expected, the sensortag consumes most of the power due to its complexity. Further power optimization can be applied to the sensor tag such as enabling duty cycle and turning on power saving mode. It should be noted that essentially, the only component added by $\mathrm{pH}$-Watch to existing wearables with pulse oximeters is the passive PANI sensor, so power overhead is just that due to software processing of $\mathrm{pH}$ values.

\section{DISCUSSION}

In this section, we address issues in $\mathrm{pH}$ Watch as well as issues pertaining to adopting our $\mathrm{pH}$ sensing approach in current day fitness trackers.

Limited evaluations. In our evaluations, we had experimented with only 10 participants (6 participants for testing the accuracy of $\mathrm{pH}$ sensing and 4 participants for assessing the realtime continuous sensing capability $\left(\mathrm{pH}+\mathrm{HR}+\mathrm{SpO}_{2}\right)$ of $\mathrm{pH}$ Watch. Currently our user-trials are limited in number and we would be conducting more user trials in future to validate the accuracy of $\mathrm{pH}$ Watch.

Improvement of $\mathrm{SpO}_{2}$ measurements. In our experiments without any motion, the addition of PANI produces a maximum error of $<3.3 \%$ in $\mathrm{SpO}_{2}$ measurements. An error of $>6 \%$ was reported during experiments with motion where random complex motion artifacts were made. $\mathrm{SpO}_{2}$ values depend on both the $\mathrm{AC}$ and $\mathrm{DC}$ part of the PPG signal. Motion artifacts affect the AC part of the PPG signal and PANI integration slightly modifies the DC part of the PPG signal. However, motion artifacts contribute to most of the $\mathrm{SpO}_{2}$ error. For instance, waving a hand up and down can cause $1.31 \%$ decrease [38] in the average accuracy with a finger pulse oximeter which is very sensitive. However, in our case, our experiments were done on wrist based pulse oximeter along with random hand motions which included rotations and complex hand movements. This error can potentially be reduced to $<5 \%$ in the future with better motion compensation algorithms, in order to meet FDA approval for $\mathrm{SpO}_{2}$ measurements. Our current prototype does not meet FDA approval. Currently, we have not evaluated our SpO2 sensing accuracy for SpO2 values between 80 to $90 \%$. All participants involved in our evaluation were healthy and they had very high $\mathrm{SpO} 2$ values above $90 \%$. In future, we need to evaluate $\mathrm{pH}$ watch for participants with low SpO2 values between 80 to $90 \%$. In $\mathrm{pH}$ watch, we only use time windows of 5 seconds with sample size of 125 PPG samples due to the hardware constraints of CC2650. This results in $\mathrm{pH}$ watch producing $\mathrm{HR}$ and $\mathrm{SpO} 2$ measurements varying by a maximum of $6.41 \%$ and $6.73 \%$ during hand motions. But this could easily be improved by using a more powerful low power MCU such as nordic nRF52832 which has $128 \mathrm{~KB}$ RAM.

Effect of sweat residue on the $\mathbf{p H}$ sensing accuracy. The residue of salts remaining on the skin after sweat evaporates should not cause any changes to the long-term $\mathrm{pH}$ sensing accuracy of $\mathrm{pH}$ Watch since $\approx 200 \mu \mathrm{L}$ of liquid sweat would be required for changes in the PANI based sensing material. During our experiments, we observed that solid residue does not cause changes to the polyaniline polymer. In the future, we will need to conduct specific experiments to assess the effect of solid residue on PANI.

Initialization of tissue DC $\left(D C^{\text {init }}\right)$. In $\mathrm{pH}$ Watch, our $\mathrm{pH}$ sensing approach relies on initialization of tissue DC component $\left(D C^{\text {init }}\right)$ without sweat which in turn requires the user to measure tissue DC component for first 3 seconds every time before exercise. Such manual initialization can be readily automated in today's smart watches such as Fitbit which has the "SmartTrack" [39] feature for automatic exercise recognition which always runs in the background. This auto exercise recognition can initialize $D C^{\text {init }}$ using the first $3 \mathrm{~s}$ of PPG signal once exercise is detected. Also, we require users to remain stationary during DC initialization to obtain a very accurate DC initialization devoid of motion artifacts. However, we have not considered people with diabetes which might cause changes in the DC initialization due to blood sugar changes.

Future research on detecting risk of dehydration. Although we have only reported how real-time $\mathrm{pH}$ values could benefit in detecting dehydration during exercise, the ultimate future aim of our research is to empower current day fitness trackers with highly reusable, real-time dehydration detection from sweat. In future, this research study would encourage researchers to study patterns between heart rate and $\mathrm{pH}$ measurements to predict risk of dehydration from human sweat, thereby enabling athletes to keep themselves rehydrated from time to time.

\section{CONCLUSION}

In this work, we have successfully demonstrated our wearable prototype - $\mathrm{pH}$ Watch for low power, highly reusable and real-time monitoring of $\mathrm{pH}$ values from sweat using pulse oximeters that are increasingly found in most of the current day fitness trackers. Our ultimate goal is to enable molecular biomarker sensing on ubiquitous wearables.

\section{ACKNOWLEDGMENTS}

We thank the shepherd Xia Zhou and the anonymous reviewers for their insightful comments. This research was partially funded by Singapore National Research Foundation: NRF-RSS2016-005. This work was also supported in part by funding from NUS Research Scholarship, MOE, NMRC, NUS BIGHEART, A*STAR IMCB Independent Fellowship and NUS Early Career Research Award.

\section{REFERENCES}

[1] M-H Schmid-Wendtner and Hans Christian Korting. "The pH of the skin surface and its impact on the barrier function". In: Skin pharmacology and physiology 19.6 (2006), pp. 296-302.

[2] RM Morgan, MJ Patterson, and MA Nimmo. "Acute effects of dehydration on sweat composition in men during prolonged exercise in the heat”. In: Acta Physiologica Scandinavica 182.1 (2004), pp. 37-43.

[3] Mark J Patterson, Stuart DR Galloway, and Myra A Nimmo. "Variations in regional sweat composition in normal human males". In: Experimental physiology 85.6 (2000), pp. 869-875.

[4] Fitbit. Fitbit versa specifications. 2018. uRL: https://www.fitbit.com/shop/versa.

[5] Garmin. Garmin Fenix 5X pulse oximeter. 2018. URL: https://www8.garmin. $\mathrm{com} / \mathrm{manuals}$ / webhelp / fenix5plus / EN - US / GUID - 4D425925 - D4EE - 4C26 B974-5375D0670860.html. 
[6] Gašper Slapničar and Mitja Luštrek. "Blood Pressure Estimation with a Wristband Optical Sensor". In: Proceedings of the 2018 ACM International foint Conference and 2018 International Symposium on Pervasive and Ubiquitous Computing and Wearable Computers - UbiComp 18 (2018). DOI: 10.1145/3267305. 3267708

[7] Ashutosh Sabharwal and Ashok Veeraraghavan. "Bio-Behavioral Sensing: An Emerging Engineering Area”. In: GetMobile: Mobile Computing and Communications 21.3 (2017), pp. 11-18.

[8] Zhilin Zhang, Zhouyue Pi, and Benyuan Liu. "TROIKA: A general framework for heart rate monitoring using wrist-type photoplethysmographic signals during intensive physical exercise". In: IEEE Transactions on biomedical engineering 62.2 (2015), pp. 522-531.

[9] A Koh et al. "A soft, wearable microfluidic device for the capture, storage, and colorimetric sensing of sweat. Science translational medicine". In: Science translational medicine 8.366 (2016), 366ra165.

[10] D Morris et al. "Bio-sensing textile based patch with integrated optical de tection system for sweat monitoring”. In: Science translational medicine 139.1 (2009), pp. 231-236.

[11] Michele Caldara et al. "A wearable sensor platform to monitor sweat $\mathrm{pH}$ and skin temperature”. In: Body Sensor Networks (BSN), 2013 IEEE International Conference on. IEEE. 2013, pp. 1-6.

[12] Michele Caldara et "Optical monitoring of sweat $\mathrm{pH}$ by a textile fabric wearable sensor based on covalently bonded litmus-3 glycidoxypropyltrimethoxysilane coating". In: Sensors and Actuators B Chemical 222 (2016), pp. 213-220.

[13] Vincenzo F Curto et al. "Real-time sweat $\mathrm{pH}$ monitoring based on a wearable chemical barcode micro-fluidic platform incorporating ionic liquids". In: Sensors and Actuators B: Chemical 171 (2012), pp. 1327-1334

[14] Ahyeon Koh et al. "A soft, wearable microfluidic device for the capture, storage, and colorimetric sensing of sweat". In: Science Translational Medicine 8.366 (2016), 366ra165-366ra165

[15] Hnin Yin Yin Nyein et al. "A wearable electrochemical platform for nonin vasive simultaneous monitoring of Ca2+ and pH”. In: ACS nano 10.7 (2016) pp. 7216-7224.

[16] Seung Yun Oh et al. "Skin-Attachable, Stretchable Electrochemical Sweat Sen sor for Glucose and pH Detection". In: ACS applied materials \& interfaces 10.16 (2018), pp. 13729-13740.

[17] Wenting Dang et al. "Stretchable wireless system for sweat $\mathrm{pH}$ monitoring". In: Biosensors and Bioelectronics 107 (2018), pp. 192-202.

[18] Bandodkar AJ et al. "Tattoo-based potentiometric ion-selective sensors for epidermal pH monitoring". In: Analyst 138.1 (2013), pp. 123-128.

[19] Tamaki Matsumoto, Takahisa Ushiroyama, and Noriyuki Tatsumi. "Lower pe ripheral circulation in eumenorrheic young women with premenstrual symptoms”. In: BioPsychoSocial Medicine 1 (2007), pp. 1-9. ISSN: 17510759. DOI: 10. 1186/1751-0759-1-8.

[20] Maxim Integrated. MAX30101-High-Sensitivity Pulse Oximeter and Heart-Rate Sensor for Wearable Health. URL: http://datasheets.maximintegrated.com/en/ ds/MAX30101.pdf.
[21] Openhak. Better understand activity tracking. URL: https:// www . openhak. com/.

[22] Hexiwear. Hexiwear - Wearable and IoT development kit. 2017. URL: https:// www.hexiwear.com/.

[23] W Gao and J Song. "Polyaniline film based amperometric $\mathrm{pH}$ sensor using a novel electrochemical measurement system". In: Electroanalysis: An International fournal Devoted to Fundamental and Practical Aspects of Electroanalysis 21.8 (2009), pp. 973-978.

[24] Jo Hee Yoon et al. "High performance flexible $\mathrm{pH}$ sensor based on polyaniline nanopillar array electrode". In: Journal of colloid and interface science 490 (2017), pp. 53-58.

[25] UW Grummt et al. "Polyaniline based optical pH sensor". In: Analytica chimica acta 357.3 (1997), pp. 253-259.

[26] Larisa Florea et al. "Dynamic $\mathrm{pH}$ mapping in microfluidic devices by integrating adaptive coatings based on polyaniline with colorimetric imaging techniques". In: Lab on a Chip 13.6 (2013), pp. 1079-1085.

[27] Liangfang Zhu et al. "Formyl-Modified Polyaniline for the Catalytic Dehydration of Fructose to 5-Hydroxymethylfurfural". In: ChemSusChem 9.16 (2016), pp. 2174-2181.

[28] Lee $\mathrm{H}$ et al. "A graphene-based electrochemical device with thermoresponsive microneedles for diabetes monitoring and therapy”. In: Nature nanotechnology 11 (2016), pp. 566-72.

[29] Narayanaswamy R Kuswandi B. "Polymeric encapsulated membrane for optrodes". In: Fresenius' journal of analytical chemistry 364 (1999), pp. 605-7.

[30] Jiaxing Huang et al. "Polyaniline nanofibers: facile synthesis and chemical sensors". In: Journal of the American Chemical Society 125.2 (2003), pp. 314-315.

[31] Andrea Rinaldi et al. "A Flexible and Highly Sensitive Pressure Sensor Based on a PDMS Foam Coated with Graphene Nanoplatelets”. In: Sensor 16.12 (2016), p. 2148

[32] Lídia Tiggemann et al. "Low-cost gas sensors with polyaniline film for aroma detection". In: Journal of Food Engineering 180 (2015), pp. 16-21.

[33] Neyer. A Fleger. M. "PDMS microfluidic chip with integrated waveguides for optical detection". In: Microelectronic engineering 83 (2006), pp. 1291-1293.

[34] Vree TB, Muskens AT, and Van Rossum JM. "Excretion of amphetamines in human sweat". In: (1972).

[35] Edward D Chan, Michael M Chan, and Mallory M Chan. "Pulse oximetry: understanding its basic principles facilitates appreciation of its limitations". In: Respiratory medicine 107.6 (2013), pp. 789-799.

[36] Jaqub Ghairat and Hassan Mouhsen. "Motion artifact reduction in PPG signals". In: (2015).

[37] Meir Nitzan, Ayal Romem, and Robert Koppel. "Pulse oximetry: fundamentals and technology update". In: Medical Devices (Auckland, NZ) 7 (2014), p. 231.

[38] Wei-Jheng Lin and Hsi-Pin Ma. "A physiological information extraction method based on wearable PPG sensors with motion artifact removal". In: 2016 IEEE International Conference on Communications (ICC). IEEE. 2016, pp. 1-6.

[39] Fitbit. SmartTrack. URL: https://www.fitbit.com/smarttrack. 